\title{
$\mathrm{XVI}$. The relation of circular polarization, as occurring both in the amorphous and crystalline states, to the symmetry and partitioning of homogeneous structures, i.e. of crystals
}

\section{William Barlow}

To cite this article: William Barlow (1897) XVI. The relation of circular polarization, as occurring both in the amorphous and crystalline states, to the symmetry and partitioning of homogeneous structures, i.e. of crystals, Philosophical Magazine Series 5, 43:261, 110-117, DOI: $10.1080 / 14786449708620967$

To link to this article: http://dx.doi.org/10.1080/14786449708620967

\section{Published online: 08 May 2009.}

Submit your article to this journal ¿

\section{Article views: 2}

Q View related articles 5 
XVI. The Relation of Circular Polarization, as occurring both in the Amorphous and Crystalline States, to the Symmetry and Partitioning of Homogeneous Structures, i. e. of Crystals. By WilidaM Barlow*.

THE general prevalence of the belief that circular polariza1 tion is a property connected with the symmetrical arrangement of small parts of the bodies displaying it, dates from Reusch's famous device for obtaining it with a stack of mica plates arranged as a staircase spiral. As we are now acquainted with the nature of the repetition in space which constitutes homogeneity of structure, and know how structures thus formed may be partitioned symmetrically $\dagger$, the time seems to have arrived for comparing the various distinct classes of cases in which circular polarization occurs with the geometrical possibilities for homogeneous structures, unbroken and broken, i.e. for substances both in the crystalline and the fluid conditions.

Adopting substantially the classification given by Pope, in a paper which he has recently published $\ddagger$, we may place the substances which possess the powerr of converting plane-polarized light into circularly-polarized light under five heads.

1. Those which exhibit circular polarization only in the amorphous state (that is, when dissolved, melted, or converted into gas).

2. Those among the substances showing circular polarization in the crystalline state only which owe the property to complex grouping or intercalation of crystal individuals, such as occurs in many cases of pseudo-symmetry.

3. Those other substances showing circular polarization in the crystalline state only in which it is an inherent property of the homogeneous structure itself.

4. Those among the substances which rotate the plane of polarization both when amorphous and when erystalline which owe the property, as displayed in the solid state, to complex intercalation of individual crystals.

5. Those other substances which possess the property both in the amorphous and in the crystalline state in which the circular polarization, when the latter state prevails, is a specific property of the homogeneous structure, and is not due to intercalation.

On the threshold of an inquiry as to the relation between

* Communicated by the Author.

† 'Mineralogical Magazine,' 1896, xi. p. 119.

$\ddagger$ Trans. Chem. Soc. 1896, p. 971. 
circular polarization and structure the very obvious conclusion suggests itself that since circular pularization is essentially an enantiomorphous property, if it is traceable to arrangement of parts, the structures in which it oucurs must be enantiomorphous, $i . e$. not identical with their own mirrorimages.

Now if we adopt the conclusion, reached in my paper just referred to, that the partitioning which achieves the separation of a crystal into individual molecules must be one which is compatible with the coincidence-movements (Deckbewegungen) of the structure ${ }^{*}$, it is evident that, when a structure identical with its own mirror-image undergoes such a partitioning, the fragments produced will either themselves be identical with their own mirror-images, or will occur in equal numbers in two kinds which are enantiomorphs. And that in the former case there will be no enantiomorphism, and that in the latter the effect of such a property of one kind of fragment will, so far as the general mass is concerned, be just cancelled by the sinilar property, of opposite hand, of the other kind of fragment.

We see therefore that we may confine our attention to those types of homogeneous structure which are enantiomorphs, not only when dealing with crystals, but also when considering those cases where the substances displaying the property in question are in one of the amorphous states.

If circular polarization is in some way connected with structure, the following general principles may be laid down :-

I. Circular polarization is due to the existence of certain arrangements of parts or particles of a structure, and these arrangements, which will be designated effective configurations, are of enantiomorphous form.

II. Absence of the property in a substance may be due either to the absence of effective configurations, or to the presence in equal numbers of two opposite kinds, whose effects just cancel one another, the arrangement of parts displayed by one kind being generally enantiomorphous, or nearly so, to that presented by the other kind.

Let us now endeavour to arrive geometrically at a classification of homogeneous structures according to the effecive configurations which they contain, both when unbroken and when dislocated, which shall be parallel to the classification given above of substances possessing the property of circular polarization.

* Min. Mag. 1896, xi. p. 130.

$\mathrm{K} 2$ 


\section{Class 1.}

As a parallel to those substances which exhibit circular polarization in the amorphous state only, we bave

Enantiomorphous homogeneous structures which while unbroken contain effective configurations of two opposite hands whose effects just cancel one another, but which, after being symmetrically partitioned and dislocated, contain but one kind, the other being destroyed in the process of dislocation.

An example from the cubic system will make this clear.

A homogeneous structure with gyrohedral symmetry of type $8 *$ is, as we know, not identical with its own mirrorimage ; and if such a structure contains effective configurations, we may suppose that they consist of particles so placed as to form a single kind of Sohncke's 24-point-set, the centre A of the configurations occupying, therefore, the centres of half the cubes of a close-packed system of cubes filling space, symmetrically chosen, i.e. so chosen that they are in contact at their edges only + .

But it can easily be shown that particles thus placed may equally well be regarded as forming other 24-point-sets whose centres lie at the centres B of the cubes of the other half of the system of cubes.

And if the distance of a particle from the point A nearest to it is the same, or practically the same, as its distance from the nearest point $\mathrm{B}$, the only material difference between the forms of the 24-point-sets thus related will be that one will be righthanded the other left-handed.

If therefore the parts or particles of the effective configurations occupy positions thus about midway between the two kinds of singular points $\ddagger A, B$, the rotation produced by their arrangement about centres $A$ may be neutralized by the effect of their arrangement, of the contrary band, about centres B.

The homogeneous structure, when in the solid or unbroken state, will then, as a whole, produce practically no rotation.

Not so, however, if it is symmetrically partitioned into fragments having one or other of the two kinds of singular points A, B for their centres, and then dislocated.

For it is evident that this will destroy one of the two kinds of effective configurations, and leave the other to pro-

* Zoitschr.f. Kryst. 1894, xxiii. p. 18.

† The centres A form therefore a "cubisches flächencentrirtes Baumgitter," or, as Sohncke calls it, a " regulär oltaëdrisches Raumgitter."

i Zeitschr, f. Kryst. xxiii. p. 60. 
duce a rotation which is not counterbalanced by one of the opposite hand.

Points thus capable of being regarded as simultaneously forming two sets of distinct contigurations which are enantiomorphous to one another or nearly so, are easily found in either of the enantiomorphous types in which all the coincidence-movements taken together are identical with their ovn mirror-images, i.e. in all of these types except Nos. 3, 4, 14, $15,16,17,18,19,21,22,26,27,30,31,32,33,42,43,44$, 45,46 , and 47 .

In the last-named types the presence of helical structures of one hand without the helical structures enantiomarphous to them seems to prohibit any arrangement leading to certain compensation.

\section{Class 2.}

Parallel to those among the substances showing circular polarization in the crystalline state only which owe the property to complex grouping or intercalation of crystal individuals, such as occurs in many cases of pseudo-symmetry, we have

Homogeneous structures, single individuals of which contain no effective configurations, but which, when differently-orientated twin individuals of them are intercalated, form such configurations where the twin individuals meet, but not in two kinds which are enuntiomorplis.

An example illustrating this may be presented by a twin combination of type $48 *$.

For suppose that two identical homogeneous structures of this enantiomorphous type, which contain no effective configurations, are so intercalated that while their orientations about an axis differ by $60^{\circ}$, one particular set of axes is common to both $\dagger$, and further that at least one set of the singular points on these axes in one individual form, with the corresponding set in the other individual, a single continuous space-lattice. There are two ways in which this may happen, either the axes of one individual may have the same, or they may have the opposite orientation to that of the axes of the other individual.

- Zeitschr.f. Kryst. xxiii. p. 31.

t A case in which the system of axes taken alone always possesses higher symmetry than the structure to which it belongs has been selected. Where this is not the case, for twinning of the nature described to occur, higher symmetry of the system of axes will have to subsist as a special condition, e.g. for individuals possessing rhombic symmetry when twinning thus to have common axes, the axes, taken alone, must be $\mathrm{s} r$ situated as to form a system in trigonal or hexagonal symmetry. 
For convenience of description let us suppose the axes to be vertical.

Then wherever one of the specified singular points of the one individual is found next to and vertically over a corresponding singular point of the other individual, the parts of one individual will be found turned through $60^{\circ}$ as compared with the corresponding parts of the other, and thus they will be related like two succeeding steps of a spiral staircase. And we may suppose that such a disposition, in the case in question, is an effective configuration.

And since the individual structures thus related are identical, the effective configurations produced in this way will be all identical, and no configuration enantiomorphous to them will be present to set up circular polarization of the opposite hand to neutralize that which they set up.

As the formation of the effective configurations is an incident of the crystal grouping, they will not be found when the structure is partitioned and dislocated, but only where the twinning competent to produce them exists.

\section{Class 3.}

The parallel to the case of those of the substances showing circular polarization in the crystalline state only in which the property is inherent, is found in

Homogeneous structures which contain effective configurations that are not counterbalanced by configurations $\epsilon$ nantiomorphous to them, and in which, when partitioned and dislocated, these configurations are wanting, their destruction having been brought ahout by the dislocation.

An example to illustrate this case can be found in any enantiomorphous type which contains helical structure, the more prominent examples being furnished by those types in which the coincidence-movements due to the presence of belical structure are all of one hand.

For the helical structures may be supposed to be effective configurations, and when symmetrical partitioning and dislocation of the similar fragments take place, they will necessarily disappear *.

If, with the helical structure, all enantiomorphism disappears, so that the fragments of the enantiomorphous structure are, when taken alone, identical with their own mirror-images, it is evident that when a homogeneous structure is reformed from the fragments, it may be either a righthanded or a left-handed enantiomorph.

* See Min. Mag. 1896, xi. p. 133. 
With this may be compared such a fact as that sodium chlorate, which is optically inactive in solution, can be óbtained in a dextro- or lævo-rotatory form at will.

\section{Class 4.}

To those among the substances that rotate the plane of polarization both when amorphous and when crystalline which owe the property, as displayed in the solid state, to complex intercalation of individual crystals, we find a parallel in

Homogeneous structures which, like those of Class 1, while unbroken contain effective configurations of two opposite kinds whose effects just cancel one another, but which, after being symmetrically partitioned and dislocated, contain but one kind, the other being destroyed in the process of dislocation; and which also like those of Class 2, when differently orientated twin individuals of them are intercalcated, form additional effective configurations where the twinned individuals meet, but not in two kinds which are enantiomorphs.

Type $49^{*}$ will furnish an example of this if the following conditions obtain :-

$a$. The particles which furnish the effective configurations in the amorphous state, i.e. after partitioning and dislocation have taken place, form enantiomorphous 6-point-sets whose centres in the unbroken structure are a set of the principal singular points, $i$. e. of those lying at the intersections of trigonal and digonal axes.

b. These particles are so placed that the 6-point-sets of opposite band which, in the unbroken structure, they also form + , and whose centres constitute another set of principal singular points, are practically enantiomorphous to the firstnamed 6-point-sets, and the circular polarization produced by one set of 6-point-sets neutralizes, on the whole, in the unbroken structure, that of opposite rotation produced by the other set.

c. Intercalation of twinned individuals, similar to that described as occurring in Class 2, produces, as in that case, effective configurations where the individuals meet.

The origin of the circular polarization displayed in the amorphous state is in this class different from that to which it is traceable in the twinned condition.

A new and striking instance of this class of circular polarization is given by Pope in the paper already referred to $\ddagger$, viz., trans- $\pi$-camphotricarboxylic acid.

* Zeitschr. f. Kryst. xxiii. p. 31.

+ Comp. p. 112.

† Trans. Chem. Soc. 1896, pp. 972 and 978. 


\section{Class 5.}

Finally, a parallel to those among the substances which rotate the plane of polarization both when amorphous and when crystalline, in which the circular-polarization, when the latter state prevails, is a specific property of the homogeneous structure, is afforded by

Homogeneous stmctures which contain effective configurations that do not, on the whole, neutralize one another, and some of which are not destroyed when the structure is partitioned symmetrically and dislocated.

A careful examination of the varions types of enantiomorphous homogeneous structure reveals the fact that in most of them the presence of effective configurations of any one kind, which are not helical structures, involves the presence in the same structure of other configurations formed of the same particles as those which compose the first-named configurations but differently allotted, which configurations, whether very similar or not, are of the opposite hand*.

In such cases the two kinds of configurations need not, of course, neutralize one another, although probably in many cases they would practically do so.

However, there are many other types whose parts are not thas balanced, and which certainly will not by any such property be made inactive.

It is one of these latter which is selected as an illustration.

In a structure of type $12 \nmid$ we may suppose that the effective configurations consist of particles so placed as to form Sobncke 24-point-sets whose centres $A$ are the cube-centres of a closepacked system of cubes.

These same particles may then equally well be regarded as forming 24-point-sets whose centres B occupy all the cube angles, but the two kinds of configuration cannot possibly in this case be supposed to neutralize one another because they are both of the same hand. They must, on the contrary, be expected to reinforce one another.

Symmetrical partitioning into similar fragments, followed by dislocation, if it leaves one of the two sets of configurations intact, will destroy the other, and thas we may look for different specific rotation in the broken from that in the unbroken structure as the result of such a dislocation.

Cases are conceivable in which effective configurations of opposite hand are present in a structure, and those destroyed

* Comp. p. 112.

$\dagger$ Zeitschr. f. Kryst. xxiii. p. 21. 
by dislocation are the dominant ones. In this event the sign of the specific rotation will change as we pass from the unbroken to the broken condition.

Wyroubuff's discovery that in the case of rubidium tartrate, which is optically active in both states, the sense of the rotation is not the same in both, may be mentioned in this connexion*.

We see from the foregoing that exactly corresponding to the five classes into which substances that possess the power of converting plane-polarized light into circularly-polarized light can be divided, there are five classes of structure distinguishable by characteristic geometrical features.

It can therefore hardly be doubted that circular polarization is a mechanical effect depending on the relative situation of the ultimate parts of bodies, and that the disappearance of the property and the changes in it observed when the state of a body displaying it alters are also mechanical effects entirely due to changes in geometrical configuration.

XVII. The Transitive Substitution Groups of Order 8p, p being any Prime Number. By G. A. Miller, Ph.D. $\dagger$

$T^{N}$ a recent paper published in this Journal $\ddagger$ we determined 1 all the possible operation groups of order $8 p$. The present paper is devoted to the more general problem of determining all the possible transitive substitution groups of this order. In solving this problem it is convenient to employ the results of the preceding paper together with the following two theorems.

THEOREM I.-The number of transitive substitution groups which are simply isomorphic to an operation group $(G)$ is equal to the number of different systems of subgroups of $(G)$, such that each system includes all the subgroups that are transformed into each other when $\mathrm{G}$ is transformed by all the operations that are commutative to it, and none of these systems includes any invariant (self-conjugate) subgroup of $\mathrm{G}$ with the exception of identity. The substitutions of these simply isomorphic transitive groups can be directly obtained from $\mathrm{G}$, and the degrees of these groups are the quotients obtained by dividing the order of G by the orders of the subgroups in the given systems $\$$.

* Journ. de Physique, 1894, p. 451.

+ Communicated by the Author.

I August 1896, vol. xlii. pp. 195-200.

Cf. Dyck, Mathematische Annalen, rol. xxii. p. 90. 